\title{
Orthotopic transplantation of retinoblastoma cells into vitreous cavity of zebrafish for screening of anticancer drugs
}

Dong Hyun Jo ${ }^{1,2,3+}$, Dain Son ${ }^{4 \dagger}$, Yirang Na ${ }^{4}$, Manyoung Jang ${ }^{5}$, Jae-Hoon Choi ${ }^{5}$, Jin Hyoung Kim ${ }^{1,2}$, Young Suk Yu ${ }^{1,6}$, Seung Hyeok Seok ${ }^{4^{*}}$ and Jeong Hun Kim ${ }^{1,2,3,6^{*}}$

\begin{abstract}
Background: With high throughput screening, novel therapeutic agents can be efficiently identified. Unfortunately, researchers only resort to in vitro cell viability assays for screening of anticancer drugs for retinoblastoma, the most common intraocular cancer in the childhood. Current available animal models of retinoblastoma require more than 2 weeks for tumour formation and the investigation of the efficacy of therapeutic agents. In this study, we established a novel orthotopic transplantation model of retinoblastoma in zebrafish as an in vivo animal model for screening of anticancer drugs.

Methods: We injected retinoblastoma cells into the vitreous cavity of zebrafish at 48 hours after fertilization. Eyeballs of zebrafish were scanned daily under the confocal laser microscope, and the tumor population was quantitatively analyzed by measuring the mean intensity of green fluorescent protein (GFP). Transplanted retinoblastoma cells were isolated to perform further analyses including Western blotting and reverse transcriptase-polymerase chain reaction to confirm that retinoblastoma cells maintained their characteristics as tumor cells even after transplantation and further isolation. To figure out the potential of this model for screening of anticancer drugs, zebrafish were cultured in Ringer's solution containing carboplatin and melphalan after the injection of retinoblastoma cells.
\end{abstract}

Results: The degree of the tumor population was dependent on the number of retinoblastoma cells injected and maintained stably for at least 4 days. Transplanted retinoblastoma cells maintain their proliferative potential and characteristics as retinoblastoma cells after isolation. Interestingly, systemic application of carboplatin and melphalan demonstrated significant reduction in the tumor population, which could be quantitatively analyzed by the estimation of the mean intensity of GFP.

Conclusions: This orthotopic retinoblastoma model in zebrafish is expected to be utilized for the screening of anticancer drugs for the treatment of retinoblastoma.

Keywords: Anticancer drug screen, Orthotopic transplantation, Retinoblastoma, Zebrafish

\footnotetext{
* Correspondence: lamseok@snu.ac.kr; steph25@snu.ac.kr

${ }^{\dagger}$ Equal contributors

${ }^{4}$ Department of Microbiology and Immunology and Institute of Endemic

Disease, College of Medicine, Seoul National University, Seoul, Republic of

Korea

${ }^{1}$ Fight against Angiogenesis-Related Blindness (FARB) Laboratory, Clinical Research Institute, Seoul National University Hospital, Seoul, Republic of Korea

Full list of author information is available at the end of the article
} 


\section{Background}

For the effective treatment of cancer, it is crucial to select proper regimens of anticancer drugs, which are based on the robust development of various regimens to improve efficacy and minimize toxicity. Last few decades are blossomed with the introduction of novel therapeutics such as targeted therapy or immunotherapy for cancers $[1,2]$. In the pediatric cancers, there are also many attempts for the introduction and actual uses of novel therapeutics [3]. Unfortunately, that was not the case in the treatment of retinoblastoma, the most common intraocular malignancy in childhood but an uncommon disease of the incidence of $1 / 20,000$ births worldwide [4,5]. Currently, carboplatin-based regimens are widely utilized in the systemic chemotherapy and melphalan is commonly employed in the intraarterial chemotherapy, which addresses the tumor by the administration of anticancer drugs to ophthalmic artery via catheterization [6,7]. Although these approaches have yielded satisfactory clinical outcomes, there are still patients who are compelled to undergo enucleation, the complete removal of the eyeball, resulting in irreversible vision loss for the lifetime.

Novel therapeutic agents can enhance the efficacy of currently utilized administration modalities including intravenous, intraarterial, and intravitreal injection [8]. For the development and screening of novel therapeutic agents, the effective screening tools are desperately required. As for retinoblastoma, a previous attempt on multiple screening of anticancer drugs simply utilized in vitro cell viability assays and measurements of chemosensitivities in the human tumor clonogenic assay using primary retinoblastoma cells and established cell lines [9]. However, there is no effective in vivo animal model for multiple screening of anticancer drugs at one time. Currently available animal models including mice with genetic aberrations and murine orthotopic transplantation models require more than 2 weeks to form tumors; therefore, they are not suitable for rapid and high throughput screening of anticancer drugs $[10,11]$.

In this study, we transplanted retinoblastoma cells into the vitreous cavity of zebrafish to establish a novel orthotopic transplantation model of retinoblastoma in zebrafish that can be utilized for high throughput screening of anticancer drugs. Zebrafish are suitable for extensive testing of multiple drugs because of relatively low maintenance cost, accessibility of in vivo imaging, and tiny size $[12,13]$. Especially, we investigated the potential of orthotopic transplantation of retinoblastoma to mimic the tumor microenvironment as much as possible. In addition, we can quantitatively analyze the degree of the tumor population in this model with a public image processing program. Furthermore, there is no change in the characteristics of tumor cells between before and after the injection, demonstrating the stability of transplanted cells as tumor cells during the study. Also, we identified the possibility of this model as a screening tool for various anticancer drugs with 2 widely utilized anticancer drugs for retinoblastoma, carboplatin and melphalan.

\section{Results}

Human retinoblastoma cells are injected into the vitreous cavity of zebrafish embryo at $\mathbf{4 8} \mathrm{hpf}$

To establish the orthotopic transplantation model of retinoblastoma in zebrafish, we injected retinoblastoma cells into the vitreous cavity of zebrafish embryo at 48 hours post fertilization (hpf) (Figure 1A). Normally, the larval pigment pattern of zebrafish was developed completely within 6 days after fertilization [14]; therefore Nphenylthiourea (PTU) was used 1 day after fertilization to inhibit pigmentation of the eyeballs of zebrafish to maximize the visibility of the tumor population under the confocal laser microscope. PTU of the concentration utilized in this study $(0.2 \mathrm{mM})$ did not show inhibitory effect on the proliferation of retinoblastoma cells by itself (data not shown). In zebrafish, the vitreous cavity is a bowl-like hollow space between the lens and the retina. When the cells were adequately injected into this space, they could spread along the vitreous cavity and maintain their population. As shown in Figure 1B, retinoblastoma cells showed stable maintenance in the vitreous cavity of zebrafish 2 days after the injection (days post injection, dpi).

The degree of the tumor population is dependent on the number of retinoblastoma cells injected

To figure out the potential of the quantification of the data in this novel zebrafish model of retinoblastoma, we intravitreally injected retinoblastoma cells, SNUOT-Rb1 cells which we previously established [15], of different
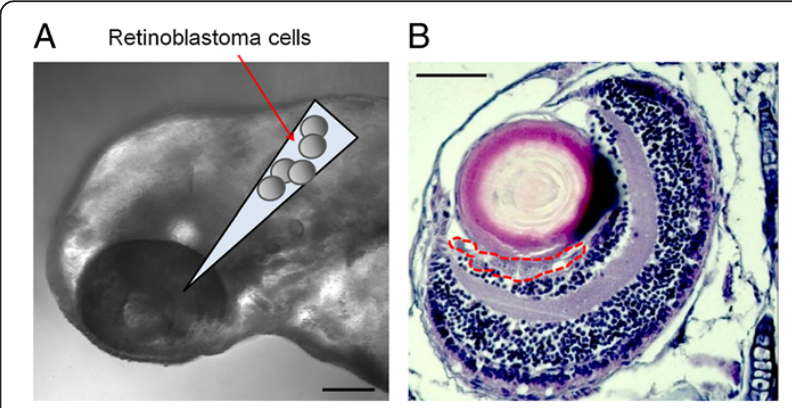

Figure 1 Human retinoblastoma cells are injected into the vitreous cavity of zebrafish embryo at $\mathbf{4 8} \mathbf{~ h p f}$. Via glass capillaries attached to the microinjector with the pneumatic pump system, retinoblastoma cells are injected into the vitreous cavity of zebrafish embryo (A). 4- $\mu \mathrm{m}$ thin paraffin section demonstrates the existence of retinoblastoma cells 2 days after the injection in the vitreous cavity, the bowl-like hollow space between the lens and the retina (B). Scale bar, $100 \mu \mathrm{m}$. 
numbers, 20 and 100 cells (Figure $2 \mathrm{~A}$ and $\mathrm{B}$ ). To reduce variations between injections, we performed injection on over 200 zebrafish at a sitting with the pneumatic pump system. With the aid of microinjectors with glass capillaries and the regulator of injection pressure in the pneumatic pump system, we could minimize the intraexperiment and inter-experiment variations. For the quantification of the data, the mean intensity of green fluorescent protein (GFP) expression was measured using ImageJ software.

As expected, the degree of the tumor population was determined by differential amount of retinoblastoma cells injected (Figure 2C). The mean intensity of GFP expression was selected as the representative value for the tumor population instead of the total area of GFP expression in that the total area only represented the 2-dimensional distribution of tumor cells, not the 3-dimensional constitution of them. Interestingly, the degree of the tumor population was maintained from $1 \mathrm{dpi}$ to $4 \mathrm{dpi}$ (Figure $2 \mathrm{C}$ ). The stable maintenance of the tumor population and the differential population according to the number of injected cells are 2 important characteristics that make up the potential of this method of orthotopic transplantation of retinoblastoma cells as an animal model of retinoblastoma.

\section{Transplanted retinoblastoma cells are successfully} isolated and maintain their characteristics as tumor cells As shown in Figure 2C, the tumor population was maintained from $1 \mathrm{dpi}$ to $4 \mathrm{dpi}$. This phenomenon may be due to the stability of this model system; however, it can be due to the alteration of the characteristics of tumor cells after the transplantation into the vitreous cavity. To verify that the transplanted cells retained their characteristics as retinoblastoma cells, we isolated retinoblastoma cells at 4 dpi from the zebrafish. Because we transduced GFP containing lentiviral particles into the cells before transplantation, we could identify transplanted cells by confirming the expression of GFP. Furthermore, the selection media containing puromycin was utilized throughout the isolation process to selectively isolate transplanted retinoblastoma cells. As expected, the morphology of

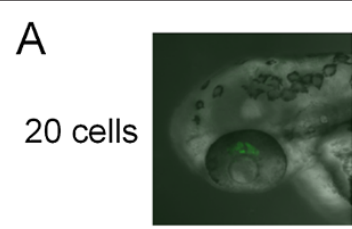

0 dpi

B

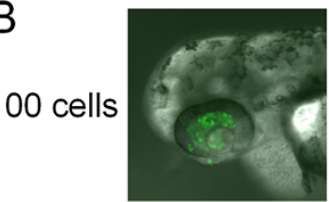

0 dpi

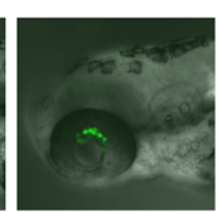

$1 \mathrm{dpi}$

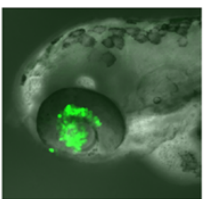

$1 \mathrm{dpi}$

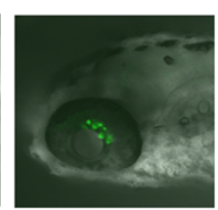

$2 \mathrm{dpi}$

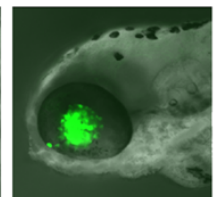

$2 \mathrm{dpi}$

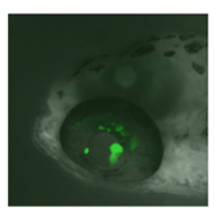

3 dpi

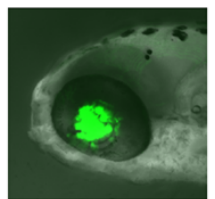

3 dpi

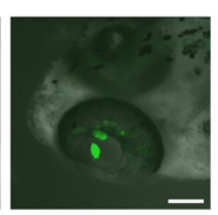

$4 \mathrm{dpi}$

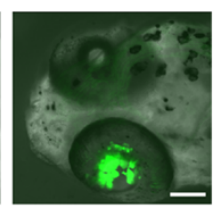

$4 \mathrm{dpi}$
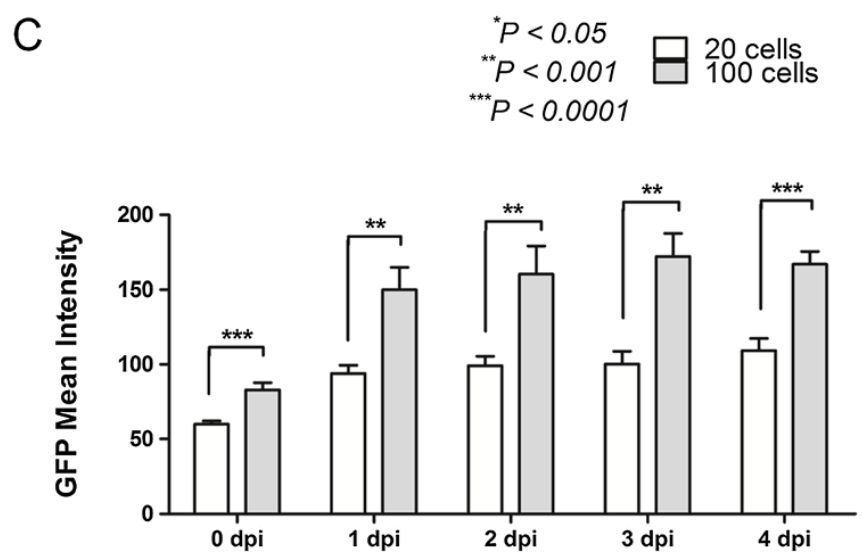

Figure 2 The degree of the tumor population is dependent on the number of retinoblastoma cells injected. We injected 20 and 100 retinoblastoma cells into the vitreous cavity of zebrafish, and the eyeballs of zebrafish embryos were scanned daily through the confocal laser microscope 20 cells, (A; 100 cells, B). Scale bar, $100 \mu \mathrm{m}$. The tumor population was quantitatively analyzed as the mean intensity of GFP expression at 4 dpi with the public image processing program, ImageJ (C). Data of 8 zebrafish per group from at least 2 independent experiments. dpi, day(s) post injection; GFP, green fluorescent protein. 
retinoblastoma cells did not change with transplantation and further isolation (Figure $3 \mathrm{~A}$ and $\mathrm{B}$ ). To demonstrate the maintenance of previously reported biochemical characteristics of SNUOT-Rb1 cells after the transplantation and isolation [15], we performed Western blot for glial fibrillary acidic protein (GFAP, glial differentiation marker) and neuron-specific enolase (NSE, neuronal differentiation marker), demonstrating no change between SNUOT-Rb1 cells and isolated cells (Figure 3C). These patterns demonstrated that transplanted cells retained the characteristics of blastoma cells that shared glial and neuronal differentiation markers. The expression of cellular retinaldehyde-binding protein (CRALBP), the marker of retinal constituent cells, also exhibited no definite change between 2 types of cells (Figure 3D).
The potential of formation of the tumor population was examined by reinjecting isolated cells into the vitreous cavity of zebrafish. Similarly with the first injection (Figure 3E), reinjection of retinoblastoma cells effectively induced the tumor population (Figure 3F). Further cultivation of isolated cells showed that the cells retained unique characteristics of SNUOT-Rb1 cells of adherent and rapid growth.

\section{A novel orthotopic retinoblastoma model in zebrafish can be utilized for the screening of anticancer drugs}

We established this orthotopic retinoblastoma model in zebrafish with the purpose of utilizing for the screening of anticancer drugs. Carboplatin and melphalan were selected

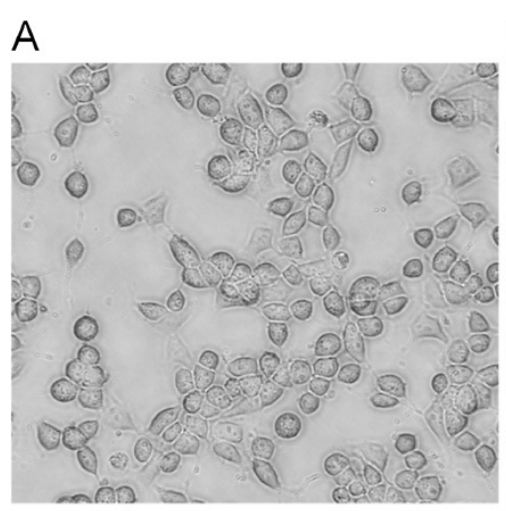

\section{B}

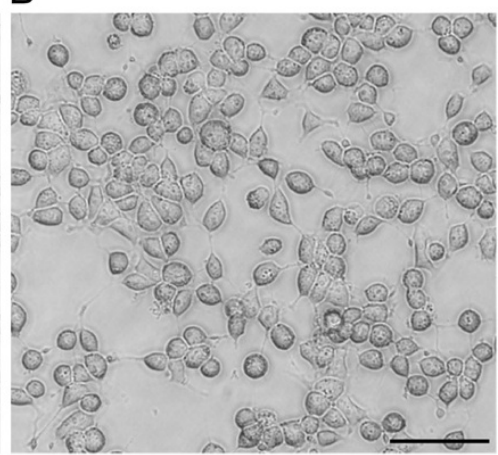

C

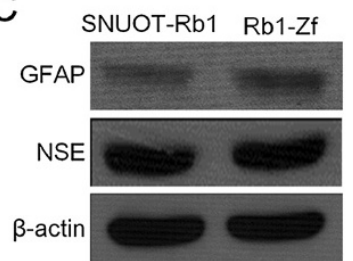

D

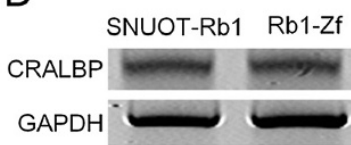

$E$

F
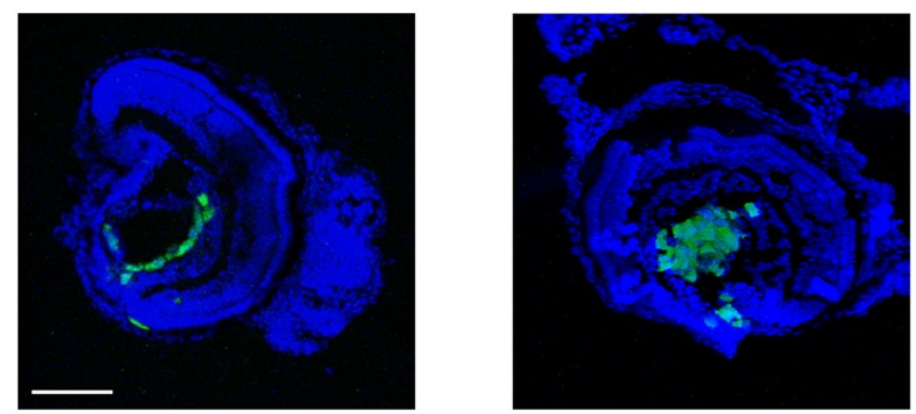

Figure 3 Transplanted retinoblastoma cells are successfully isolated and maintain their characteristics as tumor cells. Transplantation and further isolation of retinoblastoma cells do not induce morphological changes in retinoblastoma cells Before injection, (A; After transplantation and isolation, B). Scale bar, $100 \mu \mathrm{m}$. Western blotting for glial differentiation marker, GFAP and neuronal differentiation marker, NSE demonstrates no definite change between preinjected and isolated retinoblastoma cells (C). RT-PCR shows stable expression of CRALBP gene, a marker for retinal constituent cells (D). As with the first injection of retinoblastoma cells, reinjection of isolated cells demonstrates the stable tumor population 2 days after the injection First injection, (E; Reinjection of isolated cells, F). Scale bar, 100 um. Rb1-Zf, isolated cells from zebrafish into which SNUOT-Rb1 cells were injected. 
as representative drugs in this study, because carboplatin is one of the main anticancer drugs in the regimens for systemic chemotherapy [7], and melphalan is widely utilized in the regimen for intraarterial chemotherapy, which aims at the control of primary tumor or subretinal and vitreous seedings [16,17]. The treatment of retinoblastoma cells with carboplatin and melphalan for 48 hours induced reduction in the cell viability by $74 \%$ and $61 \%$ at the concentration of $200 \mu \mathrm{M}$, respectively (Figure 4A). As we observe in the clinical settings, these therapeutic agents showed inhibitory effect on proliferation of SNUOT-Rb1 cells.
Similarly with in vitro antiproliferative effect, 2 anticancer drugs demonstrated to reduce the tumor population in zebrafish (Figure $4 \mathrm{~B}$ and $\mathrm{C}$ ). To demonstrate anticancer activity of both drugs, zebrafish were cultured in Ringer's solution containing $200 \mu \mathrm{M}$ anticancer drugs right after the injection of 100 retinoblastoma cells. As shown in Figure $4 \mathrm{~B}$ and $\mathrm{C}$, therapeutic agents seemed to induce dispersion of retinoblastoma cells, not joining as a tumor in Figure 2B. We also compared the mean intensity of GFP expression between the control and the treatment groups. As expected, carboplatin and melphalan reduced the

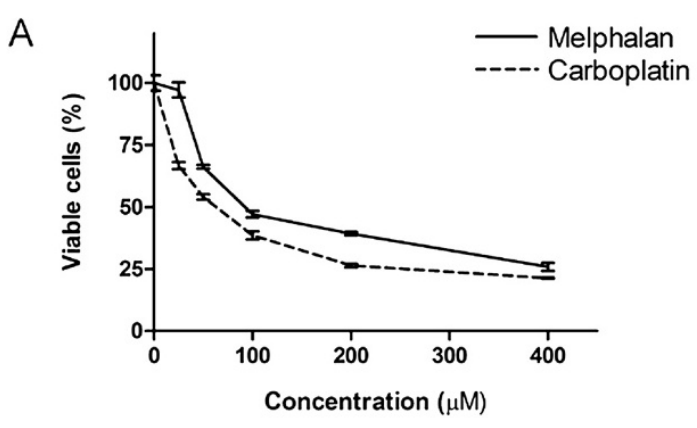

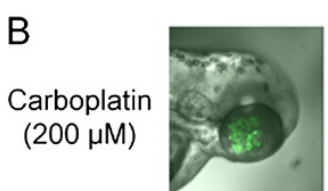

0 dpi

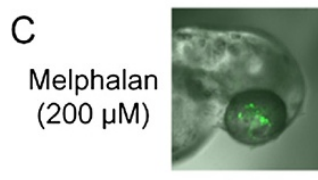

0 dpi

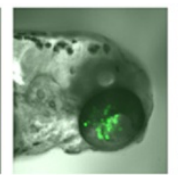

$1 \mathrm{dpi}$

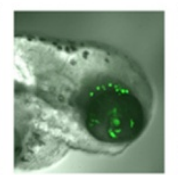

$1 \mathrm{dpi}$

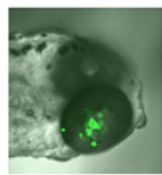

$2 \mathrm{dpi}$

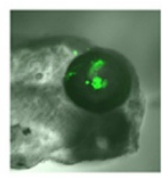

2 dpi

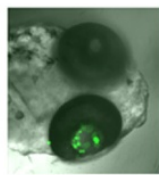

3 dpi

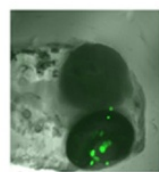

3 dpi

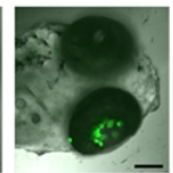

4 dpi

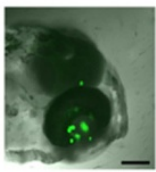

$4 \mathrm{dpi}$
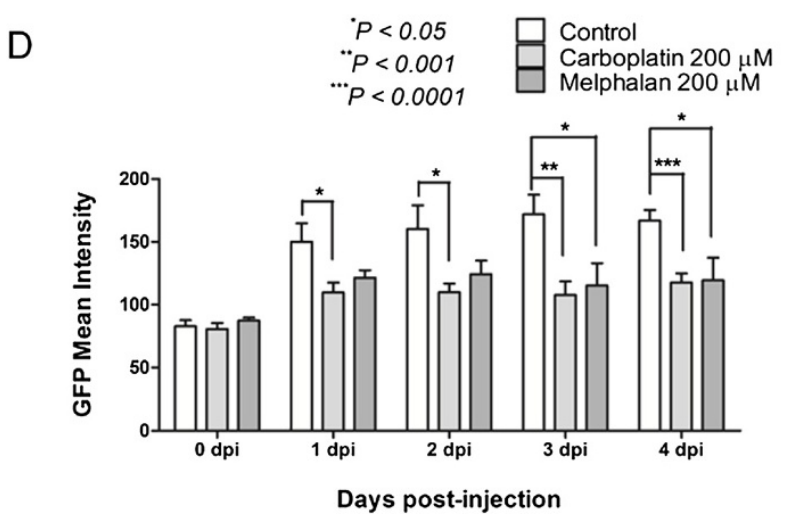

Figure 4 A novel orthotopic retinoblastoma model in zebrafish can be utilized for screening of anticancer drugs. Retinoblastoma cells are treated with carboplatin and melphalan of various concentrations from 25 to $400 \mu \mathrm{M}$ (A). Systemic application of both anticancer drugs diluted in fresh Ringer's solution induces reduction of the tumor population in zebrafish Carboplatin treatment, (B; Melphalan treatment, C). Scale bar, $100 \mu \mathrm{m}$. Quantitative analysis of the tumor response in this model yields stable results (D). Data of 8 zebrafish per group from at least 2 independent experiments. dpi, day(s) post injection; GFP: green fluorescent protein. 
expression of GFP at 4 dpi by $30 \%(\mathrm{P}<0.0001)$ and $28 \%$ $(\mathrm{P}=0.0312)$, respectively, compared to the control (Figure 4D). Like in these examples of carboplatin and melphalan, anticancer drugs can be screened in this novel orthotopic transplantation model for retinoblastoma in zebrafish.

\section{Discussion}

The development of novel effective regimens is one of the best parts in the treatment of cancer. In addition, the investigation into proper administration options is required in localized tumors such as retinoblastoma, which originates from the inner layers of the retina and extends into the adjacent structures such as vitreous cavity, optic nerve, and choroid [18,19]. In this regard, researchers on the treatment of retinoblastoma have struggled to find novel therapeutic agents and modalities to deliver anticancer drugs to tumors. Particularly, current studies are mainly focused on the development and validation of novel therapeutic modalities such as intraarterial and intravitreal chemotherapy plus conventional intravenous chemotherapy [8]. With these efforts, tumors of group $\mathrm{A}$ to $\mathrm{C}$ according to the International Classification of Retinoblastoma (ICR) demonstrate the treatment success rate of over $90 \%$ by intravenous chemotherapy with carboplatin, vincristine, and etoposide [6]; intraarterial chemotherapy with melphalan can be utilized as salvage treatment after chemoreduction failure or primary treatment for advanced stages, group D or E according to the ICR [8]. However, studies on the novel therapeutic agents for retinoblastoma slightly lag behind compared to rapid increase in the development and introduction of targeted therapy or immunotherapy in other pediatric cancers [3]. Still, enucleation is the last choice for treatment failure with chemotherapy of various administration modalities; therefore, we speculated that the introduction of novel anticancer drugs might improve the clinical outcomes of current available modalities, minimizing the risk of irreversible vision and eyeball loss.

For the development of novel therapeutic agents, multiple screening of various candidate drugs is required. Once several candidate drugs are selected from in vitro experiments, adequate in vivo studies should be performed for identification of better drugs in animal models. However, current animal models of retinoblastoma are not suitable for high throughput screening of drugs in that tumor is formed after more than 2 weeks and we cannot perform experiments on many mice at one time. For example, the orthotopic transplantation model in mice requires 4 to 8 weeks to demonstrate the efficacy of anticancer drugs, although the tumor formation is evident in all the pups [10]. Similarly, researchers wait 2 weeks after the birth to form tumors in a genetic mouse model of retinoblastoma (Chx10-Cre;Rb(lox/lox);p107(-/-);p53(lox/lox)) [11].
In this study, we completed 1 round of experiments within 1 week after the fertilization and performed injection of retinoblastoma cells into the vitreous cavity on more than 200 zebrafish at a sitting. Transplantation of tumor cells to zebrafish has been utilized for investigation of tumor biology [20,21]. In addition, a few attempts explored the possibility of zebrafish models as tools for screening of anticancer drugs [13,22,23]. In these models, tumor cells from oral squamous cell carcinoma and leukemia cell lines are injected through yolk sac. We attempted the different approach from these studies in that we injected tumor cells into the vitreous cavity, with which retinoblastoma interacts in real patients. As shown in Figure 1, we successfully injected tumor cells into the vitreous cavity of zebrafish embryos at $48 \mathrm{hpf}$.

Interestingly, the highest increase in the GFP expression occurred between $0 \mathrm{dpi}$ and $1 \mathrm{dpi}$ and the level of GFP expression maintained stably throughout the study period, when we quantitatively analyzed the data. We speculated the presumptive reasons of this phenomenon as follows: 1) right after the injection, tumor cells might be inevitably packed. The emission of fluorophores might be interfered by other obscuring tumor cells or intraocular structures such as the lens in front of tumor cells when we observed the cells under the confocal laser microscope. 2) In this regard, after the redistribution of tumor cells from $0 \mathrm{dpi}$ to $1 \mathrm{dpi}$, the expression of GFP was increased. The more important part with the alterations of GFP expression is that there was stable expression of GFP between $1 \mathrm{dpi}$ and $4 \mathrm{dpi}$. In the normal culture condition, SNUOT-Rb1 cells double their numbers every 24 hours [14]. However, in the vitreous cavity of zebrafish and previous murine models of mice, doubling of tumor cells is not that fast [10]. The interaction between the tumor and the vitreous might be different from that between the tumor and the culture media.

The distribution patterns as well as the mean intensity of GFP expression were different according to the number of injected cells. We can observe dispersed cells when we injected only 20 cells (Figure 2A). In contrast, the injection of 100 retinoblastoma cells induced more ordered distribution of tumor cells as shown in Figures $2 \mathrm{~B}$ and 3E. So we decided to inject 100 cells for further experiments on investigating into the potential of this model for screening of anticancer drugs.

Interestingly, we found the potential of this model as a tool for multiple screening of anticancer drugs with carboplatin and melphalan. We necessarily selected these 2 currently utilized drugs because they are widely utilized in real clinical settings for retinoblastoma patients. Interestingly, both drugs demonstrated effective anticancer activity on this model. These results might provide the possibility of this model as a screening tool for the evaluation of anticancer drugs which are in the development process. 
Furthermore, as previously mentioned, we can inject more than 200 zebrafish embryos at a sitting; therefore, more than 20 different candidate drugs can be screened with 1 session of experiments. Quantitative analysis of the tumor population yielded stable and reproducible results, demonstrating the usefulness of this model. After the image was captured with the confocal laser microscope, the quantitative data can be easily obtained with the public image processing program, ImageJ, by a few clicks.

\section{Conclusions}

In this model, we performed orthotopic transplantation of retinoblastoma cells into the vitreous cavity of zebrafish. In this novel animal model of retinoblastoma, it was possible to quantitatively analyze the tumor population and the response of tumors to anticancer drugs with a public image processing program. Transplanted retinoblastoma cells retained their characteristics as tumor cells during the study period. We speculated that this novel orthotopic transplantation model for retinoblastoma in zebrafish can be a gateway for the development of anticancer drugs for retinoblastoma, one of the tumors that lag behind in the view of the development and introduction of novel therapeutic agents.

\section{Methods}

\section{Zebrafish}

Adult wild-type zebrafish, purchased from a local aquarium farm and maintained in the laboratory facility, were utilized for producing embryos by breeding. Zebrafish were raised at $28.5^{\circ} \mathrm{C}$ in alternate dark-light cycles of 13 and 11 hours, respectively. Twenty-four hours after fertilization, zebrafish embryos were placed in fresh Ringer's solution with $0.2 \mathrm{mM}$ PTU (Sigma-Aldrich, St. Louis, MO) to inhibit pigmentation of eyeballs. Care, use, and treatment of animals were done in agreement with the Association for Research in Vision and Ophthalmology for the use of animals in ophthalmic and vision research and the guidelines established by the Seoul National University Institutional Animal Care and Use Committee.

\section{Cell culture}

SNUOT-Rb1 cells, from the previously established retinoblastoma cell line by our group [15], were maintained in RPMI 1640 medium (WelGENE, Daegu, Korea) supplemented with $10 \%$ fetal bovine serum (FBS; Gibco BRL, Rockville, MD) and 1\% penicillin-streptomycin solution (Invitrogen, Carlsbad, CA) at $37^{\circ} \mathrm{C}$ in the humidified atmosphere of $95 \%$ air and $5 \% \mathrm{CO}_{2}$. The cell line underwent the authentication by DNA fingerprinting analysis with short tandem repeat markers by Korean Cell Line Bank on November 22, 2012. For the visualization of cells with GFP, SNUOT-Rb1 cells were transfected with cop GFP Control Lentiviral Particles (sc-108084; Santa Cruz
Biotechnology, Santa Cruz, CA) according to the manufacturer's instructions. To select stable clones expressing GFP, cells were maintained in the culture media containing $4 \mu \mathrm{g} / \mathrm{ml}$ puromycindihydrochloride (sc-108071; Santa Cruz Biotechnology) for 2 weeks.

\section{Intravitreal injection of retinoblastoma cells}

At 48 hpf, zebrafish embryos were anesthetized with 0.042 $\mathrm{mg} / \mathrm{ml}$ Tricaine (ethyl 3-aminobenzoate methanesulfonate; Sigma-Aldrich). Eight to 10 hours before the injection, zebrafish embryos were dechorionated if necessary. For the stable injection, zebrafish were placed on the $1.7 \%$ agarose gel containing $1 \mathrm{ppm}$ methylene blue. Then, using the Pneumatic PicoPump (PV820; World Precision Instruments, Sarasota, FL), cells of the indicated number were injected into the vitreous cavity of zebrafish embryos via glass capillaries attached to the Hamilton syringe (Hamilton Company, Reno, NV) under the stereomicroscope (Leica S6 E; Leica Microsystems, Wetzlar, Germany). Eyes of zebrafish were scanned daily on the Coverglass-Bottom dish (SPL Life Sciences, Pocheon, Republic of Korea) by the confocal laser microscope (Fluoview FV1000; Olympus, Tokyo, Japan) to record the alterations in the tumor population.

\section{Quantification of tumor population}

We obtained the images of the eyeballs of zebrafish 1 hour, 24, 48, 72, 96 hours after the injection of retinoblastoma cells with the confocal laser microscope (Fluoview FV1000, Olympus). The mean intensity of GFP expression was measured using the ImageJ program (1.46r; National Institutes of Health, Bethesda, MD) [24]. After opening the image file captured with the confocal laser microscopy in the program, the color threshold was set using the menu Image $>$ Adjust $>$ Color Threshold. Then, the area over the designated threshold (brightness 48) was selected automatically with the yellow demarcation line or by putting the 'select' button on the pop-up. The mean intensity of GFP expression was calculated by the menu Analyze >Tools $>$ Color Histogram or putting the 'RGB' button twice on the pop-up in the menu Analyze $>$ Histogram to demonstrate green histogram of the image (Different approaches were required to get the values from version to version). The values were demonstrated as 'Mean' or 'gMean' on the pop-up. Eight zebrafish were included per group for experiments.

\section{Isolation of retinoblastoma cells}

Four days after the injection of retinoblastoma cells, about 150 zebrafish were collected in $4^{\circ} \mathrm{C}$ phosphate-buffered saline supplemented with $1 \%$ penicillin-streptomycin solution (Invitrogen). Then, the zebrafish were digested with 1.5 $\mathrm{mg} / \mathrm{ml}$ collagenase from Clostridium histolyticum (SigmaAldrich) in Hank's balanced salt solution supplemented 
with 5\% FBS (Gibco BRL) and 1\% penicillin-streptomycin solution (Invitrogen) at $37^{\circ} \mathrm{C}$ for 30 minutes. The isolated cells were suspended and incubated in RPMI 1640 (WelGENE) supplemented with 10\% FBS (Gibco BRL), 1\% penicillin-streptomycin solution (Invitrogen), and $4 \mu \mathrm{g} / \mathrm{ml}$ puromycindihydrochloride (Santa Cruz Biotechnology). After the sufficient colonies were formed, we proceeded on further analyses.

\section{Immunoblot}

The cells were lysed with RIPA buffer containing a protease inhibitor (Roche, Penzberg, Germany). The lysates were centrifuged at $13,000 \mathrm{rpm}$ at $4^{\circ} \mathrm{C}$ for 20 minutes. Then, the supernatants were delivered to new micro test tubes for further processes. Equal amounts of extracted proteins from the cells were separated by electrophoresis on 7.5\% SDS-PAGE and transferred to nitrocellulose membranes (AmershamHybond ECL, GE Healthcare BioSciences, Piscataway, NJ). The membranes were incubated with anti-GFAP antibody (1:1,000, ab53554, Abcam, Cambridge, United Kingdom), anti-NSE antibody (1:1,000, \#9536, Cell Signaling Technology, Beverly, MA), and anti$\beta$-actin antibody (1:3,000, A2066, Sigma-Aldrich) at $4^{\circ} \mathrm{C}$ overnight. Then, the membranes were incubated with species-specific horseradish peroxidase-conjugated secondary antibodies (Pierce, Thermo Scientific, Waltham, MA). After the treatment of membranes with Amersham $\mathrm{ECL}^{\mathrm{Tm}}$ western blotting detection reagent (GE Healthcare Bio-Sciences), the membranes were exposed to the film (AmershamHyperfilm ECL, GE Healthcare Bio-Sciences).

\section{Reverse transcriptase-polymerase chain reaction (PCR)}

Total RNA was extracted from the cells using TRIzol (Invitrogen) according to the manufacturer's instructions. For the synthesis of cDNA, $1 \mu \mathrm{g}$ of total RNA was mixed and reverse transcribed with oligo $(\mathrm{dT})_{15}$ primer, Superscript II reverse transcriptase (Invitrogen Corp.) and dNTPs. Polymerase chain reaction (PCR) was performed with the resultant cDNA, 10X PCR buffer, 2.5 mMdNTPs, $10 \mathrm{mM}$ forward and reverse primers, DNA polymerase (Corebiosystem, Seoul, Republic of Korea), and RNAse-free water. The primers for cellular retinaldehyde-binding protein (CRALBP) were $5^{\prime}$-TGGCAAAGTCAAGAAATCAC C-3' (forward) and 5' -CGTGGACAAAGACCCTCTCA-3' (reverse) [25], and the resultant product was $313 \mathrm{bp}$. PCR was performed with denaturation in 5 minutes at $94^{\circ} \mathrm{C}$, followed by 35 cycles of 30 seconds of denaturation at $94^{\circ} \mathrm{C}, 30$ seconds of annealing at $60^{\circ} \mathrm{C}$, and 30 seconds of elongation at $72^{\circ} \mathrm{C}$. The primers for GAPDH were $5^{\prime}-\mathrm{A}$ CCACAGTCCATGCCATCAC-3' (forward) and 5' -TCC ACCACCCTGTTGCTGTA-3' (reverse), and the resultant product was $500 \mathrm{bp}$. PCR was performed with denaturation in 5 minutes at $94^{\circ} \mathrm{C}$, followed by 30 cycles of 30 seconds of denaturation at $94^{\circ} \mathrm{C}, 30$ seconds of annealing at $65^{\circ} \mathrm{C}$, and
30 seconds of elongation at $72^{\circ} \mathrm{C}$. The PCR products were electrophoresed on $1 \%$ agarose gels containing ethidium bromide in a constant $100 \mathrm{~V}$ field.

\section{Preparation and treatment of anticancer drugs}

Carboplatin (C2538) and melphalan (M2011) were purchased from Sigma-Aldrich. Zebrafish were cultured in fresh Ringer's solution containing $200 \mu \mathrm{M}$ anticancer drugs after the intravitreal injection of retinoblastoma cells. The solutions were changed every 24 hours. Eyes of zebrafishembyos were scanned daily on the CoverglassBottom dish (SPL Life Sciences) by the confocal laser microscope (Fluoview FV1000, Olympus).

\section{Cell viability assay}

Cell viability was evaluated with 2-(4-Iodophenyl)-3-(4nitrophenyl)-5-(2,4-disulfophenyl)-2H-tetrazolium (water soluble tetrazolium salt, WST-1) assay using EZ-Cytox Cell Viability Assay kit (Itsbio, Seoul, Republic of Korea) according to the manufacturer's instruction. Briefly, SNUOT-Rb1 cells were plated in 96 well plates and cultured overnight $\left(1 \times 10^{4}\right.$ cells per well). The cells were treated with anticancer drugs of different concentrations $(25,50,100,200,400 \mu \mathrm{M})$ for 48 hours. Then, the reagent from EZ-Cytox Cell Viability Assay kit was applied to each well. After 2 hours of additional incubation, 96 well plates were shaken thoroughly on the shaker for 1 minute. Absorbance was measured at $450 \mathrm{~nm}$ using the microplate reader (VersaMax, Molecular Devices, Sunnyvale, CA). To confirm the results of WST-1 assay, direct estimation of viable cells using Trypan Blue Stain (Life Technologies, Carsbad, CA) was performed after the treatment with anticancer drugs of different concentrations for 48 hours.

\section{Statistical analysis}

Differences of the values between experiments were assessed with the Student's t-test. All statistical analyses were performed using GraphPad Prism (GraphPad Software, La Jolla, CA). The mean value and the standard error of the mean were shown in figures. P-values less than 0.05 were considered as statistically significant, and $* * * ; * *$ were designated as $\mathrm{P}<0.05, \mathrm{P}<0.001, \mathrm{P}<0.0001$, respectively, in figures.

\section{Abbreviations}

CRALBP: Cellular retinaldehyde-binding protein; dpi: Day(s) post injection; FBS: Fetal bovine serum; GFAP: Glial fibrillary acidic protein; GFP: Green

fluorescent protein; hpf: Hours post fertilization; ICR: International classification of retinoblastoma; NSE: Neuron-specific enolase; PCR: Polymerase chain reaction; PTU: N-phenylthiourea.

\section{Competing interests}

The authors declare that they have no competing interests.

\section{Authors' contributions}

JHK and SHS designed the research; DHJ and DS performed the research; YN, MJ, JC, JHK and YSY contributed materials and established methods; DHJ and 
DS collected data; GHJ, DS, JHK and SHS analyzed data; and DHJ, DS, JHK and SHS wrote the paper. All authors read and approved the final manuscript.

\section{Acknowledgements}

We thank to Dr. Hyoung Oh Jun for technical help on experiments. This study was supported by the Bio-Signal Analysis Technology Innovation Program (2013-036042), the Pioneer Research Program of MEST/NRF (2013005321), the Global Core Research Center (GCRC) grant from NRF/MEST, Republic of Korea (2012-0001187), the Ministry of Education, Science and Technology, (2012-0004090) and Seoul National University College of Medicine Research Grant, Republic of Korea (800-20110049).

\section{Author details}

${ }^{1}$ Fight against Angiogenesis-Related Blindness (FARB) Laboratory, Clinical Research Institute, Seoul National University Hospital, Seoul, Republic of Korea. ${ }^{2}$ Tumor Microenvironment Research Center, Global Core Research Center, Seoul National University, Seoul, Republic of Korea. ${ }^{3}$ Department of Biomedical Sciences, College of Medicine, Seoul National University, Seoul, Republic of Korea. ${ }^{4}$ Department of Microbiology and Immunology and Institute of Endemic Disease, College of Medicine, Seoul National University, Seoul, Republic of Korea. ${ }^{5}$ Department of Life Science, College of Natural Sciences, Hanyang University, Seoul, Republic of Korea. ${ }^{6}$ Department of Ophthalmology, College of Medicine, Seoul National University, Seoul, Republic of Korea.

Received: 30 March 2013 Accepted: 3 July 2013

Published: 9 July 2013

\section{References}

1. Gan HK, Burgess AW, Clayton AH, Scott AM: Targeting of a conformationally exposed, tumor-specific epitope of EGFR as a strategy for cancer therapy. Cancer Res 2012, 72:2924-2930.

2. Hong CW, Zeng Q: Awaiting a new era of cancer immunotherapy. Cancer Res 2012, 72:3715-3719.

3. Norris RE, Adamson PC: Challenges and opportunities in childhood cancer drug development. Nat Rev Cancer 2012, 12:776-782.

4. Kim JH, Yu YS: Incidence (1991 1993) and survival rates (1991 2003) of retinoblastoma in Korea. J Korean Ophthalmol Soc 2010, 51:542-551.

5. Dyer MA, Rodriguez-Galindo C, Wilson MW: Use of preclinical models to improve treatment of retinoblastoma. PLoS Med 2005, 2:e332.

6. Shields CL, Mashayekhi A, Au AK, Czyz C, Leahey A, Meadows AT, Shields JA: The International Classification of Retinoblastoma predicts chemoreduction success. Ophthalmology 2006, 113:2276-2280

7. Shields CL, Kaliki S, Rojanaporn D, Al-Dahmash S, Bianciotto CG, Shields JA: Intravenous and intra-arterial chemotherapy for retinoblastoma: what have we learned? Curr Opin Ophthalmol 2012, 23:202-209.

8. Shields CL, Fulco EM, Arias JD, Alarcon C, Pelligrini M, Rishi P, Kaliki S, Bianciotto CG, Shields JA: Retinoblastoma frontiers with intravenous, intra-arterial, periocular, and intravitreal chemotherapy. Eye (Lond) 2012, 27:253-264.

9. Inomata $M$, Kaneko A: Chemosensitivity profiles of primary and cultured human retinoblastoma cells in a human tumor clonogenic assay. Jpn J Cancer Res 1987, 78:858-868.

10. Kim JH, Kim JH, Yu YS, Kim DH, Kim CJ, Kim KW: Antitumor activity of arsenic trioxide on retinoblastoma: cell differentiation and apoptosis depending on arsenic trioxide concentration. Invest Ophthalmol Vis Sci 2009, 50:1819-1823.

11. Nemeth KM, Federico S, Carcaboso AM, Shen Y, Schaiquevich P, Zhang J, Egorin M, Stewart C, Dyer MA: Subconjunctival carboplatin and systemic topotecan treatment in preclinical models of retinoblastoma. Cancer 2011, 117:421-434.

12. Liu S, Leach SD: Zebrafish models for cancer. Annu Rev Pathol 2011, 6:71-93.

13. Jung DW, Oh ES, Park SH, Chang YT, Kim CH, Choi SY, Williams DR: A novel zebrafish human tumor xenograft model validated for anti-cancer drug screening. Mol Biosyst 2012, 8:1930-1939.

14. Kelsh RN, Brand M, Jiang YJ, Heisenberg CP, Lin S, Haffter P, Odenthal J, Mullins MC, van Eeden FJ, Furutani-Seiki M, Granato M, Hammerschmidt M, Kane DA, Warga RM, Beuchle D, Vogelsang L, Nüsslein-Volhard C: Zebrafish pigmentation mutations and the processes of neural crest development. Development 1996, 123:369-389.
15. Kim JH, Kim JH, Yu YS, Kim DH, Kim CJ, Kim KW: Establishment and characterization of a novel, spontaneously immortalized retinoblastoma cell line with adherent growth. Int J Oncol 2007, 31:585-592.

16. Ghassemi F, Shields CL: Intravitreal melphalan for refractory or recurrent vitreous seeding from retinoblastoma. Arch Ophthalmol 2012, 130:1268-1271.

17. Shields $C L$, Bianciotto CG, Jabbour P, Ramasubramanian A, Lally SE, Griffin GC, Rosenwasser R, Shields JA: Intra-arterial chemotherapy for retinoblastoma: report No. 1, control of retinal tumors, subretinal seeds, and vitreous seeds. Arch Ophthalmol 2011, 129:1399-1406.

18. Dyer MA, Bremner R: The search for the retinoblastoma cell of origin. Nat Rev Cancer 2005, 5:91-101.

19. Shields CL, Shields JA, Baez K, Cater JR, De Potter P: Optic nerve invasion of retinoblastoma. Metastatic potential and clinical risk factors. Cancer 1994, 73:692-698.

20. Nicoli S, Ribatti D, Cotelli F, Presta M: Mammalian tumor xenografts induce neovascularization in zebrafish embryos. Cancer Res 2007, 67:2927-2931.

21. Taylor AM, Zon LI: Zebrafish tumor assays: the state of transplantation. Zebrafish 2009, 6:339-346.

22. Corkery DP, Dellaire G, Berman JN: Leukaemia xenotransplantation in zebrafish-chemotherapy response assay in vivo. Br J Haematol 2011, 153:786-789.

23. Pruvot B, Jacquel A, Droin N, Auberger P, Bouscary D, Tamburini J, Muller M, Fontenay M, Chluba J, Solary E: Leukemic cell xenograft in zebrafish embryo for investigating drug efficacy. Haematologica 2011, 96:612-616.

24. Schneider CA, Rasband WS, Eliceiri KW: NIH Image to ImageJ: 25 years of image analysis. Nat Methods 2012, 9:671-675.

25. Schlunck G, Martin G, Agostini HT, Camatta G, Hansen LL: Cultivation of retinal pigment epithelial cells from human choroidal neovascular membranes in age related macular degeneration. Exp Eye Res 2002, 74:571-576.

doi:10.1186/1476-4598-12-71

Cite this article as: Jo et al:: Orthotopic transplantation of retinoblastoma cells into vitreous cavity of zebrafish for screening of anticancer drugs. Molecular Cancer 2013 12:71.

\section{Submit your next manuscript to BioMed Central and take full advantage of:}

- Convenient online submission

- Thorough peer review

- No space constraints or color figure charges

- Immediate publication on acceptance

- Inclusion in PubMed, CAS, Scopus and Google Scholar

- Research which is freely available for redistribution

Submit your manuscript at www.biomedcentral.com/submit
C Biomed Central 